\title{
THE CONVEX SCATTERING SUPPORT IN A BACKGROUND MEDIUM*
}

\author{
STEVEN KUSIAK ${ }^{\dagger}$ AND JOHN SYLVESTER ${ }^{\ddagger}$
}

\begin{abstract}
We discuss inverse problems for the Helmholtz equation at fixed energy, specifically the inverse source problem and the inverse scattering problem from a medium or an obstacle. In [S. Kusiak and J. Sylvester, Comm. Pure Appl. Math., 56 (2003), pp. 1525-1548], we introduced the convex scattering support of a far field, a set which will be a subset of the convex hull of the support of any source or scattering inhomogeneity which can produce it.

We extend these results and modify the methods to locate a source within a known inhomogeneous background medium, or a deviation from that medium, using observations of a single far field. We also describe some numerical examples that illustrate the robustness of the method.
\end{abstract}

Key words. inverse scattering, Helmholtz equation, partial differential equations

AMS subject classifications. $81 \mathrm{U} 40,74 \mathrm{~J} 25,65 \mathrm{~N} 21$

DOI. $10.1137 / \mathrm{S} 0036141003433577$

1. Introduction. We study an inverse problem for the Helmholtz equation at fixed energy. Our aim is to deduce the location of the source or scatterer from observations of scattered waves made at a distance, which are called far fields. Typically, one has access to several far fields. For the inverse medium problem, the index of refraction is uniquely determined by the full scattering kernel, i.e., the observed scattered field for every possible incident wave. In special cases $[9,5,6]$, substantial information about the support of the scatterer has been obtained from the scattered field of a few, or even only one, incident wave.

In [8], we showed that, in a homogeneous background medium, we could associate the convex scattering support with a single far field. This set is the smallest convex set which supports a source that can produce that far field. We also produced a test, the circular Paley-Wiener theorem, for computing the convex scattering support in two dimensions. In [10], we introduced a different numerical method, called the range test, for computing this support in a two-dimensional homogeneous medium.

Our work was motivated by the linear sampling method of Colton and Kirsch (see [2]). They first developed a Picard test, which determines whether a far field belongs to the range of the (compact) scattering operator, as a tool for inverse scattering. This method, and the subsequent factorization method of Kirsch [7], differ from what we present here in that they require much more data (the full scattering map) and compute much more (the exact support of the scatterer).

In section 2 of this paper, we introduce the necessary scattering formalism and restate the circular Paley-Wiener theorem as a Picard test. This restatement, though less explicit, generalizes directly to inhomogeneous media and higher dimensions.

In section 3 we produce this general test. The general test tells us if a far field could have been produced by a source or a scatterer located within a specific domain,

*Received by the editors August 21, 2003; accepted for publication (in revised form) March 5, 2004; published electronically January 27, 2005. This work was supported by NSF grant DMS0099838.

http://www.siam.org/journals/sima/36-4/43357.html

${ }^{\dagger}$ Department of Applied Mathematics, University of Washington, Seattle, WA 98195 (kusiak@ amath.washington.edu).

${ }^{\ddagger}$ Department of Mathematics, University of Washington, Seattle, WA 98195 (sylvest@math. washington.edu). The research of this author was supported by ONR grant N00014-93-1-0295. 
but not whether the true source was located there. Section 4 addresses this issue by extending the concept of convex scattering support to inhomogeneous media. The conclusion is that we can locate a smallest convex set, which must be contained in the convex hull of the support of any source which radiates that far field. Conversely, we produce a source, supported in any neighborhood of the convex scattering support which does radiate that far field.

Section 5 discusses the relationship between the support of a scatterer, rather than a source, and the convex scattering support. In this case we show that the convex scattering support provides a lower bound for the convex hull of the scatterer. Unlike the source case, we don't expect this lower bound to be optimal.

Section 6 contains a description of an explicit algorithm and some numerical results. Maybe the most important observation in this section is that the practical implementation of the algorithm is much simpler and more robust than the theorem guarantees.

2. Far fields in a homogeneous medium. We model the time harmonic wave radiated by a source in a homogeneous medium as a solution to the inhomogeneous Helmholtz equation:

$$
\left(\Delta+k^{2}\right) u(x)=f(x), \quad x \in \mathbb{R}^{n} .
$$

Equation (2.1) has a unique outgoing solution, $u=G_{0}^{+} f$, which can be computed by the limiting absorption principle (see, e.g., [11, p. 147]).

$$
\begin{aligned}
G_{0}^{+} f & =\lim _{\epsilon \downarrow 0}\left(\Delta+(k-i \epsilon)^{2}\right)^{-1} f \\
& =-\lim _{\epsilon \downarrow 0} \int_{\mathbb{R}^{n}} \frac{e^{i\langle x, \xi\rangle} \widehat{f}(\xi)}{|\xi|^{2}-(k-i \epsilon)^{2}} d \xi .
\end{aligned}
$$

The limiting absorption principle chooses the unique solution $u$ of (2.1) which extends to be holomorphic in $\{\operatorname{Im}(k) \leq 0\}$ and is continuous up to the boundary. According to (one of the many theorems called) the Paley-Wiener theorem, this solution is the Fourier transform of the unique solution $\tilde{u}$ of the wave equation which is zero in the past. That is,

$$
u(k, x)=\int_{0}^{\infty} e^{-i k t} \tilde{u}(x, t) d t .
$$

We call a function in the range of $G_{0}^{+}$outgoing. We shall refer to a function as incoming if $v=G_{0}^{-} f$, where

$$
G_{0}^{-} f=\lim _{\epsilon \downarrow 0}\left(\Delta+(k+i \epsilon)^{2}\right)^{-1} f .
$$

Alternatively, $u=G_{0}^{+} f$ may be characterized as the unique solution of (2.1) satisfying the Sommerfeld radiation condition:

$$
\lim _{r \rightarrow \infty} r^{\frac{n-1}{2}}\left(\partial_{r} u-i k u\right)=0, \quad r=|x| .
$$

Inverting the Fourier transform in (2.2), we may also represent $u=G_{0}^{+} f$ (cf. [4]) as

$$
\left(G_{0}^{+} f\right)(x):=-\frac{i}{4}\left(\frac{k}{2 \pi}\right)^{\frac{n-2}{2}} \int_{\mathbb{R}^{n}}|x-y|^{\frac{2-n}{2}} H_{\frac{n-2}{2}}^{(1)}(k|x-y|) f(y) d y .
$$


Here, $H_{(n-2) / 2}^{(1)}$ is the Hankel function of the first kind. The representation of $G_{0}^{-}$uses the other Hankel function; i.e., its kernel is the complex conjugate of the kernel of $G_{0}^{+}$.

The simplest estimate for the solution operators $G_{0}^{ \pm}$is on the weighted $L^{2}$ spaces, $\mathrm{H}_{\delta}^{s}\left(\mathbb{R}^{n}\right)$. For $\delta=0, \mathrm{H}_{0}^{s}\left(\mathbb{R}^{n}\right)$ is the Sobolev space, $\mathrm{H}^{s}\left(\mathbb{R}^{n}\right)$. For $\delta>0$,

$$
\|f\|_{s, \delta}=\left\|\left(1+|x|^{2}\right)^{\delta / 2} f\right\|_{s, 0} .
$$

Proposition 2.1. The operators $G_{0}^{ \pm}$are bounded as maps between the weighted $L^{2}$ spaces

$$
G_{0}^{ \pm}: \mathrm{H}_{\delta}^{s}\left(\mathbb{R}^{n}\right) \longrightarrow \mathrm{H}_{-\delta}^{s+2}\left(\mathbb{R}^{n}\right)
$$

for any real $s$ and any $\delta>\frac{1}{2}$. Moreover, $G_{0}^{-}$is the Hilbert space adjoint of $G_{0}^{+}$on $L^{2}$ (i.e., $s=0$ in (2.4)). That is,

$$
G_{0}^{+*}=G_{0}^{-} .
$$

Proof. The estimate was first proved in [1]. Once we have it, it is a simple matter to interchange the order of integration in the $L^{2}$ pairing to check that the two operators are adjoints.

The far field describes the asymptotics of $u$ as $|x| \rightarrow \infty$. Stationary phase applied to $(2.2)$ or Hankel function asymptotics applied to (2.3) yields

$$
u(x) \sim \frac{e^{i k|x|}}{|x|^{(n-1) / 2}} C_{n, k} \int_{\mathbb{R}^{n}} e^{-i k\langle\Theta, y\rangle} f(y) d y, \quad|x| \rightarrow \infty,
$$

where $\Theta=\frac{x}{|x|}$ is a unit vector on the $n-1$-dimensional sphere $S^{n-1}$ and

$$
C_{n, k}=\frac{-i}{\sqrt{8 \pi}}\left(\frac{k}{2 \pi}\right)^{\frac{n-2}{2}} e^{-i(n-1) \pi / 4} .
$$

Hence, given a source $f$ we define the far field, $u_{\infty}=F_{0} f$, by

$$
\begin{aligned}
\left(F_{0} f\right)(\Theta) & =\int_{\mathbb{R}^{n}} e^{-i k\langle\Theta, y\rangle} f(y) d y \\
& =\hat{f}(k \Theta) .
\end{aligned}
$$

The mapping properties of $F_{0}^{+}$are important for us.

Proposition 2.2. $\mathrm{F}_{0}^{+}$is a compact linear map

$$
F_{0}^{+}: \mathrm{H}_{\delta}^{s}\left(\mathbb{R}^{n}\right) \longrightarrow L^{2}\left(S^{n-1}\right) .
$$

Its adjoint with respect to the distributional (not the Hilbert space) pairing

$$
{F_{0}^{+}}^{\dagger}: L^{2}\left(S^{n-1}\right) \longrightarrow \mathrm{H}_{-\delta}^{-s}\left(\mathbb{R}^{n}\right)
$$

is the Herglotz operator

$$
\begin{aligned}
\left(F_{0}^{+\dagger} \alpha\right)(x) & =(\mathcal{H} f)(x) \\
& =\int_{S^{n-1}} e^{i k\langle\Theta, x\rangle} \alpha(\Theta) d S(\Theta) .
\end{aligned}
$$


Remark 2.3. Functions in the range of the Herglotz operator are usually referred to as incident fields. They are the $\mathrm{H}_{-\delta}^{s}$ solutions of the homogeneous (or free) Helmholtz equation for all real $s$ and any $\delta>\frac{1}{2}$. The Herglotz operator represents these free solutions as superpositions of plane waves.

Proof. The boundedness of $F_{0}^{+}$follows easily from the representation (2.7). We need only note that if $f \in \mathrm{H}_{\delta}^{s}$, then $\hat{f} \in \mathrm{H}_{s}^{\delta}$. As $\delta>\frac{1}{2}$, the restriction map from $\mathrm{H}_{s}^{\delta}\left(\mathbb{R}^{n}\right)$ to $L^{2}$ of the codimension one sphere, $S^{n-1}$, is a compact operator.

The boundedness of $F_{0}^{\dagger}$ follows from the boundedness of $F_{0}$. The equality (2.8) can be seen by using formula (2.6), pairing with an $L^{2}$ far field, and interchanging the order of integration. Nevertheless, we give a proof that relies more on scattering.

Let $u=G_{0}^{+} f$ and $v=\mathcal{H} \alpha$. Stationary phase shows that $v$ has the asymptotics

$$
v(x) \sim C_{n, k} \frac{e^{i k|x|}}{|x|^{(n-1) / 2}} \alpha(\theta)+C_{n, k} \frac{e^{-i k|x|}}{|x|^{(n-1) / 2}} \alpha(-\theta), \quad|x| \rightarrow \infty .
$$

Applying Green's theorem on the ball of radius $R$ gives

$$
\int_{\partial B_{R}} v \frac{\partial u}{\partial \nu}-\frac{\partial v}{\partial \nu} u=\int_{B_{R}} v\left(\Delta+k^{2}\right) u-\left(\Delta+k^{2}\right) v u .
$$

Letting $R \rightarrow \infty$ and making use of (2.5) and (2.9) allows us to evaluate the left-hand side of (2.10). Recalling that $v$ is a free solution of the Helmholtz equation removes the second term from the left-hand side so that

$$
\begin{aligned}
\int_{S^{n-1}} \alpha(\theta)\left(F_{0} f\right)(\theta) d S(\theta) & =\int_{B_{R}} v f \\
\left\langle\alpha, F_{0} f\right\rangle & =\langle\mathcal{H} \alpha, f\rangle .
\end{aligned}
$$

Proposition 2.2 has a more general statement which will prove convenient in the next section.

Theorem 2.4. Suppose that $s_{1}+s_{2}>-2, \delta>\frac{1}{2}$, and that $u$ and $v$ satisfy

$$
\begin{aligned}
& u \in \mathrm{H}_{-\delta}^{s_{1}+2}\left(\mathbb{R}^{n}\right) \quad \text { and } \quad\left(\Delta+k^{2}\right) u \in \mathrm{H}_{\delta}^{s_{1}}\left(\mathbb{R}^{n}\right), \\
& v \in \mathrm{H}_{-\delta}^{s_{2}+2}\left(\mathbb{R}^{n}\right) \quad \text { and } \quad\left(\Delta+k^{2}\right) v \in \mathrm{H}_{\delta}^{s_{2}}\left(\mathbb{R}^{n}\right) ;
\end{aligned}
$$

then as $|x| \rightarrow \infty$,

$$
\begin{aligned}
& u(x) \sim \frac{e^{i k|x|}}{|x|^{(n-1) / 2}} u_{\infty}^{+}(\theta)+\frac{e^{-i k|x|}}{|x|^{(n-1) / 2}} u_{\infty}^{-}(\theta), \\
& v(x) \sim \frac{e^{i k|x|}}{|x|^{(n-1) / 2}} v_{\infty}^{+}(\theta)+\frac{e^{-i k|x|}}{|x|^{(n-1) / 2}} v_{\infty}^{-}(\theta),
\end{aligned}
$$

and

$$
\left\langle u_{\infty}^{+}, v_{\infty}^{-}\right\rangle-\left\langle u_{\infty}^{-}, v_{\infty}^{+}\right\rangle=\left\langle u,\left(\Delta+k^{2}\right) v\right\rangle-\left\langle\left(\Delta+k^{2}\right) u, v\right\rangle .
$$

Remark 2.5. When we write " $\sim$ " meaning "asymptotic to," we mean classical asymptotics only in the case that $u$ happens to be smooth near infinity. This is always the case if $\left(\Delta+k^{2}\right) u$ has compact support. In the more general setting, the space of functions that satisfy (2.11) (or (2.12)) form a Hilbert space, and we are asserting that the mappings

$$
u \mapsto u_{\infty}^{ \pm}
$$


extend by continuity as mappings from that Hilbert space into $L^{2}\left(S^{n-1}\right)$.

Proof. Suppose first that $u$ and $v$ are compactly supported and smooth. Then every such $u$ (and $v$ ) is a linear combination of an outgoing function and a solution of the homogeneous Helmholtz equation, i.e., an outgoing wave plus a Herglotz function. Each of these has the asymptotics asserted in (2.5) and in (2.9), and therefore their sum had these asymptotics as well. Now apply Green's formula as in (2.10) to obtain (2.14).

Finally, notice that the left-hand side of (2.14) is a continuous bilinear functional with respect to $L^{2}$ convergence and the right-hand side is continuous when $u_{n} \rightarrow u$ and $v_{n} \rightarrow v$ in the topologies of (2.11).

DEFINITION 2.6. We shall refer to $u_{\infty}^{+}$as the outgoing far field of $u$ and $u_{\infty}^{-}$as its incoming far field. An outgoing function has zero incoming far field, i.e., $u_{\infty}^{-}=0$. A Herglotz function has outgoing and incoming far fields related by the antipodal map (2.9). Intuitively, one can see this by thinking about a spherical incoming wave passing through the origin to become an outgoing wave.

In [8], we began to study the scattering support of a far field. The first step is to ask whether a far field could have been produced by a source which is a distribution supported in a closed set. We recall that the restricting of a distribution to an open set means restricting it to act on the subspace $C_{0}^{\infty}(\Omega)$ of $C_{0}^{\infty}\left(\mathbb{R}^{n}\right)$. The support of a distribution is the closed set defined below.

DeFINITION 2.7. A point $x$ belongs to the support of a distribution $f$ if there exists no open neighborhood, $O_{x}$, such that $\left.f\right|_{O_{x}}=0$.

Distributions supported on a closed set form natural subspaces of $\mathrm{H}_{\delta}^{s}\left(\mathbb{R}^{n}\right)$.

DEFINITION 2.8. $\mathrm{H}_{0}^{s}(\Omega)$ is the closed subspace of $\mathrm{H}_{\delta}^{s}\left(\mathbb{R}^{n}\right)$ consisting of those distributions which are supported in $\bar{\Omega}$.

The definition is independent of $\delta$ as long as $\Omega$ is bounded.

We point out that this is different from $H^{s}(\Omega)$, which denotes the restrictions of distributions to a bounded open set, and is not a subspace of any $\mathrm{H}_{\delta}^{s}\left(\mathbb{R}^{n}\right)$. In fact, $\mathrm{H}^{-s}(\Omega)$ is the natural dual to $\mathrm{H}_{0}^{s}(\Omega)$ for all real $s$. For bounded open sets $\Omega$ and positive $s$, our definition of $\mathrm{H}_{0}^{s}(\Omega)$ coincides with the common definition, i.e., the closure of $C_{0}^{\infty}(\Omega)$ in the $\mathrm{H}^{s}$ norm. In [8], we proved the following theorem.

TheOREM 2.9. Let $\alpha \in L^{2}\left(S^{1}\right)$ represent a far field. There exists $f \in \mathrm{H}_{0}^{s}\left(B_{R}\right)$ such that

$$
F_{0}^{+} f=\alpha=\sum_{n=-\infty}^{\infty} \alpha_{n} e^{i n \theta}
$$

if and only if

$$
\sum_{n=-\infty}^{\infty}\left|\frac{\alpha_{n} n^{s}}{\sigma_{n}(R)}\right|^{2}<\infty
$$

where

$$
\sigma_{n}(R)=\left(\int_{0}^{R}\left|J_{n}(k r)\right|^{2} r d r\right)^{\frac{1}{2}} .
$$

In section 3 of this paper, we will prove a generalization of this result to variable index of refraction, higher dimensions, and more general domains. We close this section with a restatement of this theorem which anticipates the generalization to follow. 
We don't give the proof here, as it will follow as a corollary of the more general Theorem 3.6 in section 3.

THEOREM 2.10. Let $\alpha \in L^{2}\left(S^{n-1}\right)$ represent a far field. Let $\left.F_{0}^{+}\right|_{\mathrm{H}_{0}^{s}(\Omega)}$ represent the restriction of the compact operator $F_{0}^{+}$to $\mathrm{H}_{0}^{s}(\Omega)$ and let

$$
\left.F_{0}^{+}\right|_{\mathrm{H}_{0}^{s}(\Omega)}=\sum \sigma_{n} \psi_{n} \otimes \overline{\phi_{n}}
$$

be its singular value decomposition. Then

$$
\alpha \in \operatorname{Range}\left(\left.F_{0}^{+}\right|_{\mathrm{H}_{0}^{s}(\Omega)}\right)
$$

if and only if

$$
\sum\left|\frac{\left(\alpha, \psi_{n}\right)}{\sigma_{n}}\right|^{2}<\infty
$$

To facilitate the comparison of Theorems 2.9 and 2.10, we describe two examples with $\Omega$ equal to $B_{R} \in \mathbb{R}^{2}$, the ball of radius $R$ centered at the origin. We can separate variables in this case, representing the operator $F_{0}^{+}$in terms of complex exponentials, Bessel functions, and the characteristic function of the ball, $\chi_{B_{R}}$.

$$
\left.F_{0}^{+}\right|_{L^{2}\left(B_{R}\right)}=\sum_{n=-\infty}^{\infty} \mathrm{e}^{i n\left(\theta-\frac{\pi}{2}\right)} \otimes \chi_{B_{R}} \mathrm{e}^{-i n\left(\phi-\frac{\pi}{2}\right)} J_{n}(k r) .
$$

Because we are in $L^{2}$, its Hilbert space adjoint is

$$
\left(\left.F_{0}^{+}\right|_{L^{2}\left(B_{R}\right)}\right)^{*}=\sum_{n=-\infty}^{\infty} \chi_{B_{R}} \mathrm{e}^{i n\left(\phi-\frac{\pi}{2}\right)} J_{n}(k r) \otimes \mathrm{e}^{-i n\left(\theta-\frac{\pi}{2}\right)}
$$

and its singular values are the eigenvalues of $\left(\left.\left.F_{0}^{+}\right|_{L^{2}\left(B_{R}\right)} F_{0}^{+}\right|_{L^{2}\left(B_{R}\right)} ^{*}\right)$

$$
\sigma_{n}^{2}=4 \pi^{2} \int_{0}^{R} J_{n}^{2}(k s) s d s
$$

so we see that (2.16) and (2.17) agree in the case $s=0$.

Next, we consider $\left.F_{0}^{+}\right|_{\mathrm{H}_{0}^{1}\left(B_{R}\right)}$. The operator itself has the same representation as in (2.18); we are just considering it on a smaller subspace. A bit of a calculation shows that the Hilbert space adjoint is now

$$
\left(\left.F_{0}^{+}\right|_{\mathrm{H}_{0}^{1}\left(B_{R}\right)}\right)^{*}=\sum_{n=-\infty}^{\infty} \chi_{B_{R}} \mathrm{e}^{i n\left(\phi-\frac{\pi}{2}\right)}\left(J_{n}(k r)-\left(\frac{r}{R}\right)^{|n|} J_{n}(k R)\right) \otimes \mathrm{e}^{-i n\left(\theta-\frac{\pi}{2}\right)}
$$

with singular values (we'll call them $\tilde{\sigma}_{n}$ 's)

$$
\tilde{\sigma}_{n}^{2}=4 \pi^{2}\left[\int_{0}^{R} J_{n}^{2}(k s) s d s-\frac{J_{n}(k R)}{R^{n}} \int_{0}^{R} J_{n}(k s) s^{n+1} d s\right] .
$$

Now, the large $n$ asymptotics of the Bessel function is

$$
J_{n}(k r) \sim \frac{1}{\sqrt{\pi n}}\left(\frac{e k r}{2 n}\right)^{n} \quad \text { for } n \gg k r .
$$


Integrating with respect to $r$ then gives

$$
\sigma_{n}^{2} \sim \frac{8 \pi}{(\mathrm{e} k)^{2}}\left(\frac{\mathrm{e} k R}{2 n}\right)^{2 n+2}\left(1+O\left(\frac{1}{n}\right)\right), \quad n \rightarrow \infty,
$$

while the leading order asymptotics of the two terms in (2.19) cancel, so that

$$
\tilde{\sigma}_{n}^{2}=O\left(\frac{\sigma_{n}^{2}}{n^{2}}\right)
$$

which agrees with (2.16) in the case $s=1$.

3. Far fields in an inhomogeneous medium. If the medium is inhomogeneous, (2.1) is replaced by

$$
\left(\Delta+k^{2} n(x)\right) u(x)=f(x), \quad x \in \mathbb{R}^{n} .
$$

The coefficient $n(x)$ is the index of refraction and is the square of the reciprocal of the wave speed at $x$. We will assume that $n$ has positive imaginary part, that $n-1$ is compactly supported, and that $n \in L^{p}\left(\mathbb{R}^{n}\right)$ for $p>\max (n-2, n / 2)$. The unique continuation principle holds for this is the class of $n$ 's.

It will be convenient to rewrite (3.1) as

$$
\left(\Delta+k^{2}-q(x)\right) u=f
$$

with $q=k^{2}(n-1)$. Because $q$ is not necessarily smooth, we must restrict the regularity of the Sobolev spaces. We want to allow single and double layer potentials as sources, so that the application of our results to active scattering will include scattering from an obstacle. We will treat $f \in \mathrm{H}_{\delta}^{s-2}\left(\mathbb{R}^{n}\right)$ with $0 \leq s \leq n / p$ and $\delta>\frac{1}{2}$.

We will standardize the notation used in this section. We will use $\eta$ and $\sigma$ to denote unrestricted real numbers. The symbols $\delta, p$, and $s$ will always satisfy the inequalities

$$
\begin{gathered}
\delta>1 / 2, \\
p>\max (2, n / 2), \\
0 \leq s \leq n / p .
\end{gathered}
$$

Our next theorem asserts the existence of the analogues of $G_{0}$ and $F_{0}$.

THEOREM 3.1. Let $q \in L^{p}\left(\mathbb{R}^{n}\right)$ and have compact support. Let $f \in \mathrm{H}_{\delta}^{s-2}\left(\mathbb{R}^{n}\right)$ with $p, s, \delta$ satisfying (3.2). Then there exists a unique outgoing (resp., incoming) solution of

$$
\left(\Delta+k^{2}-q(x)\right) u=f
$$

which has the asymptotic behavior

$$
u(x) \sim \frac{e^{i k|x|}}{|x|^{(n-1) / 2}} u_{\infty}^{ \pm}(\Theta), \quad|x| \rightarrow \infty .
$$

Moreover, the unique solution $u$ is computed by the operator

$$
\begin{aligned}
u(x) & =\left(G_{q}^{ \pm} f\right)(x) \\
& :=\left(I-G_{0}^{ \pm} q\right)^{-1} G_{0}^{ \pm} f \\
& =G_{0}^{ \pm}\left(I-q G_{0}^{ \pm}\right)^{-1} f
\end{aligned}
$$


and

$$
\begin{aligned}
u_{\infty}^{ \pm}(\Theta) & =\left(F_{q}^{ \pm} f\right)(\Theta) \\
& :=F_{0}^{ \pm}\left(I-q G_{0}^{ \pm}\right)^{-1} f .
\end{aligned}
$$

Additionally, both $G_{q}^{ \pm}$and $F_{q}^{ \pm}$are compact operators:

$$
\begin{aligned}
& G_{q}^{ \pm}: \mathrm{H}_{\delta}^{s-2}\left(\mathbb{R}^{n}\right) \rightarrow \mathrm{H}_{-\delta}^{s-n / p}\left(\mathbb{R}^{n}\right), \\
& F_{q}^{ \pm}: \mathrm{H}_{\delta}^{s-2}\left(\mathbb{R}^{n}\right) \rightarrow L^{2}\left(S^{n-1}\right) .
\end{aligned}
$$

The proof requires that we show that $\left(I-G_{0}^{ \pm} q\right)$ is invertible. Let $M_{q}$ denote the operator of multiplication by $q$. Then we have the following lemma.

Lemma 3.2. Let $q$ be a compactly supported function on $\mathbb{R}^{n}$. For any real $p \geq 2$, any real $\delta$ and $\eta$, and any $0 \leq s \leq \frac{n}{p}$,

$$
M_{q}: \mathrm{H}_{\eta_{1}}^{s}\left(\mathbb{R}^{n}\right) \rightarrow \mathrm{H}_{\eta_{2}}^{s-\frac{n}{p}}\left(\mathbb{R}^{n}\right)
$$

is bounded.

Proof. According to Hölder's inequality,

$$
\begin{aligned}
\|q u\|_{L^{2}\left(\mathbb{R}^{n}\right)} & \leq\|q\|_{L^{p}\left(\mathbb{R}^{n}\right)}\|u\|_{L^{\frac{2 p}{p-2}}\left(\mathbb{R}^{n}\right)} \\
& \leq\|q\|_{L^{p}\left(\mathbb{R}^{n}\right)}\|u\|_{\mathrm{H}^{\frac{n}{p}}\left(\mathbb{R}^{n}\right)}
\end{aligned}
$$

with the second line a consequence of the Sobolev inequality. Thus

$$
M_{q}: \mathrm{H}_{0}^{\frac{n}{p}}\left(\mathbb{R}^{n}\right) \rightarrow L^{2}\left(\mathbb{R}^{n}\right)
$$

is bounded. Duality implies that

$$
M_{q}: L^{2}\left(\mathbb{R}^{n}\right) \rightarrow \mathrm{H}_{0}^{-\frac{n}{p}}\left(\mathbb{R}^{n}\right)
$$

is also bounded. Interpolation then gives (3.8) in the case that $\delta=\eta=0$. However, because $q$ is compactly supported,

$$
\left\|\left(1+|x|^{2}\right)^{\frac{\eta-\delta}{2}} q\right\|_{L^{p}\left(\mathbb{R}^{n}\right)} \leq C\|q\|_{L^{p}\left(\mathbb{R}^{n}\right)},
$$

which implies (3.8) for any $\delta$ and any $\eta$.

As a consequence, we have the following corollary.

Corollary 3.3. Let $p \geq 2,0 \leq s \leq \frac{n}{p}$, and $\delta>\frac{1}{2}$. Then

$$
G_{0}^{+} q: \mathrm{H}_{\eta}^{s}\left(\mathbb{R}^{n}\right) \rightarrow \mathrm{H}_{-\delta}^{s+2-\frac{n}{p}}\left(\mathbb{R}^{n}\right),
$$

and

$$
q G_{0}^{+}: \mathrm{H}_{\delta}^{s-2}\left(\mathbb{R}^{n}\right) \rightarrow \mathrm{H}_{\eta}^{s-\frac{n}{p}}\left(\mathbb{R}^{n}\right)
$$

is bounded. If, in addition, $p>\frac{n}{2}$, then

$$
G_{0}^{ \pm} q: \mathrm{H}_{-\delta}^{s}\left(\mathbb{R}^{n}\right) \rightarrow \mathrm{H}_{-\delta}^{s}\left(\mathbb{R}^{n}\right)
$$

are compact. 
Proof. The first statement is a direct consequence of Proposition 2.1 and Lemma 3.2 , while the second follows from the compact embedding of $\mathrm{H}_{\eta}^{s_{1}}$ in $\mathrm{H}_{\delta}^{s_{2}}$ whenever $\eta>\delta$ and $s_{1}>s_{2}$.

COROLlary 3.4. Let $q \in L^{p}\left(\mathbb{R}^{n}\right)$ with compact support and let $p, s, \delta$ satisfy (3.2). Then $\left(I-G_{0}^{+} q\right)^{-1}$ exists as a bounded linear operator from $\mathrm{H}_{-\delta}^{s}\left(\mathbb{R}^{n}\right)$ to $\mathrm{H}_{-\delta}^{s}\left(\mathbb{R}^{n}\right)$.

Proof. Corollary 3.3 implies that $\left(I-G_{0}^{+} q\right)$ is Fredholm, so we only need to show uniqueness. Suppose that $u \in \mathrm{H}_{-\delta}^{s}\left(\mathbb{R}^{n}\right)$ satisfies

$$
\left(I-G_{0}^{+} q\right) u=0 .
$$

Repeated application of (3.9) shows us that $u \in \mathrm{H}_{-\delta}^{2}\left(\mathbb{R}^{n}\right)$ and satisfies

$$
\left(\Delta+k^{2}\right) u=q u
$$

Since $u$ is outgoing, $\bar{u}$ is incoming and satisfies

$$
\left(\Delta+k^{2}\right) \bar{u}=\overline{q u}
$$

so that we may apply (2.14) to obtain the identities

$$
\begin{aligned}
2 i k\left\langle\overline{u_{\infty}^{+}}, u_{\infty}\right\rangle & =\langle\overline{q u}, u\rangle-\langle\bar{u}, q u\rangle \\
2 i k\left\|u_{\infty}^{+}\right\|_{L^{2}}^{2} & =2 i \int \operatorname{Im} q|u|^{2}
\end{aligned}
$$

which implies

$$
\left\|u_{\infty}^{+}\right\|_{L^{2}}^{2} \leq 0
$$

Thus, $u$ is an outgoing function with no far field. Rellich's lemma [3] implies that $u$ vanishes outside the support of $q$, and unique continuation then implies that $u=0$ everywhere.

Corollary 3.5. Let $q \in L^{p}\left(\mathbb{R}^{n}\right)$ with compact support and let $p, s, \delta$ satisfy (3.2). Then $\left(I-q G_{0}^{+}\right)^{-1}$ exists as a bounded linear operator from $\mathrm{H}_{\delta}^{s-2}\left(\mathbb{R}^{n}\right)$ to $\mathrm{H}_{\delta}^{s-2}\left(\mathbb{R}^{n}\right)$.

Proof. $\left(I-q G_{0}^{+}\right)^{-1}$ is also Fredholm, so only uniqueness need be checked. Suppose $f=q G_{0}^{+} f$. Then $u=G_{0}^{+} f$ satisfies (3.10), and therefore it must be zero. However, $f=\left(\Delta+k^{2}\right) u$, so $f$ must also vanish.

Proof of Theorem 3.1. We have shown that formulas (3.3), (3.4), and (3.5) make sense. Both (3.6) and (3.7) follow from the previous corollaries and the mapping properties of $F_{0}$ and $G_{0}$. To verify that (3.3) and (3.4) are equal, we start with the factorization

$$
\left(I-G_{0}^{ \pm} q\right) G_{0}^{ \pm}=G_{0}^{ \pm}\left(I-q G_{0}^{ \pm}\right) .
$$

Now, both $\left(I-G_{0}^{ \pm} q\right)$ and $\left(I-q G_{0}^{ \pm}\right)$are invertible, so that

$$
\left(I-G_{0}^{ \pm} q\right)^{-1}\left(I-G_{0}^{ \pm} q\right) G_{0}^{ \pm}\left(I-q G_{0}^{ \pm}\right)^{-1}=\left(I-G_{0}^{ \pm} q\right)^{-1} G_{0}^{ \pm}\left(I-q G_{0}^{ \pm}\right)\left(I-q G_{0}^{ \pm}\right)^{-1}
$$

which implies

$$
G_{0}^{ \pm}\left(I-q G_{0}^{ \pm}\right)^{-1}=\left(I-G_{0}^{ \pm} q\right)^{-1} G_{0}^{ \pm}
$$


With Theorem 3.1 in place, we may extend the Picard test (2.17) to the inhomogeneous equation.

THEOREM 3.6. Let $\alpha \in L^{2}\left(S^{n-1}\right)$ represent a far field and let $\left.F_{q}^{+}\right|_{\mathrm{H}_{0}^{s}(\Omega)}$ represent the restriction of the compact operator $F_{q}^{+}$to $\mathrm{H}_{0}^{s}(\Omega)$. Then

$$
\alpha \in \operatorname{Range}\left(\left.F_{q}^{+}\right|_{\mathrm{H}_{0}^{s}(\Omega)}\right)
$$

if and only if

$$
\sum\left|\frac{\left(\alpha_{n}, \psi_{n}\right)}{\sigma_{n}}\right|^{2}<\infty
$$

where

$$
\left.F_{q}^{+}\right|_{\mathrm{H}_{0}^{s}(\Omega)}=\sum \sigma_{n} \psi_{n} \otimes \overline{\phi_{n}}
$$

is the singular value decomposition of $\left.F_{q}^{+}\right|_{\mathrm{H}_{0}^{s}(\Omega)}$.

This theorem tells us that we can look for a source in a known inhomogeneous background by simply replacing $F_{0}$ by $F_{q}$ in the convergence test given in Theorem 2.10. Of course, to apply it we must numerically or analytically compute the singular value decomposition of $\left.F_{q}^{+}\right|_{\mathrm{H}_{0}^{s}(\Omega)}$.

4. The convex scattering support. We are ready to use Theorem 3.6 to locate the support of a source in an inhomogeneous medium. In Theorem 5.2 of section 5 , we will locate the region where a medium differs from a known background by applying this test. In both cases our data will be a single far field.

As we pointed out in [8], a single far field is not enough information to uniquely determine the support of a source. For example, if $\phi$ has compact support, then $f_{\phi}=\left(\Delta+k^{2}\right) \phi$ will always have zero far field. We can always add $f_{\phi}$ to a source to produce a new one with bigger support which produces the same far field. Thus we cannot associate with a far field a set which contains the support of any source which produces it.

However, we can determine a unique smallest convex set which must be a subset of the convex hull of the support of any source which produces that far field. We refer to this set as the convex scattering support of a far field. We will show below that the convex scattering support of any nonzero far field is a nonempty closed set, and that there always exists an $L^{2}$ source, supported in an arbitrarily small neighborhood of the convex scattering support, which will reproduce the far field.

We begin with the definition.

DEFINITION 4.1. The convex scattering support of the far field $u_{\infty}$, with respect to the background $q$, is

$$
\operatorname{cS}_{k} \operatorname{supp}_{q} u_{\infty}=\bigcap_{\substack{F_{q} f=u_{\infty} \\ f \in \mathrm{H}_{\delta}^{s}\left(\mathbb{R}^{n}\right)}} \operatorname{ch}(\operatorname{supp} f) .
$$

Here, $\operatorname{ch}(\operatorname{supp} f)$ denotes the convex hull of the support of $f$.

We must take $s>-2$ because $F_{q}$ is only defined for such sources. The next lemma asserts that the $\mathrm{CS}_{k} \operatorname{supp}_{q} u_{\infty}$ doesn't depend on $s$ for $-2<s \leq 0$. It doesn't depend on $s$ at all if $q$ is smooth. We will use the notation $N_{\epsilon}(\Omega)$ to denote an open epsilon neighborhood of a set $\Omega$. 
Lemma 4.2. For any $f \in \mathrm{H}_{0}^{s}(\Omega)$ and any $\varepsilon>0$, there exists $\tilde{f} \in L^{2}\left(N_{\varepsilon}(\Omega)\right)$ such that

$$
F_{q}^{+} \tilde{f}=F_{q}^{+} f .
$$

Proof. Let $u=G_{q}^{+} f$ and let $\phi \in C^{\infty}\left(\mathbb{R}^{n}\right)$ satisfy

$$
\phi= \begin{cases}1, & x \in \mathbb{R}^{n} \backslash N_{\varepsilon}(\Omega), \\ 0, & x \in N_{\varepsilon}(\Omega),\end{cases}
$$

and set

$$
\tilde{f}=\left(\Delta+k^{2}-q(x)\right) \phi u .
$$

Now, $G_{q}^{+} f=u$ outside $N_{\varepsilon}(\Omega)$ and therefore has the same far field. Note that $\phi u$ is supported outside $\operatorname{supp} f$ so that $u$ is $\mathrm{H}^{2}$ there, and thus $\tilde{f} \in L^{2}$.

THEOREM 4.3. For any far field $\alpha \in L^{2}\left(S^{n-1}\right)$ with a compactly supported source, and any $\varepsilon>0$, there exists an $L^{2}$ source $f_{\varepsilon}$ such that $G_{q}^{+} f_{\varepsilon}=\alpha$ and

$$
\operatorname{ch}\left(\operatorname{supp} f_{\epsilon}\right) \subset N_{\epsilon}\left(\operatorname{cS}_{k} \operatorname{supp}_{q} \alpha\right) .
$$

We shall need two lemmas for the proof.

Lemma 4.4. Suppose supp $f_{1} \subset \Omega_{1}$, supp $f_{2} \subset \Omega_{2}$, and that $\mathbb{R}^{n} \backslash\left(\Omega_{1} \cup \Omega_{2}\right)$ is connected and contains a neighborhood of $\infty$. If

$$
F_{q}^{+} f_{1}=F_{q}^{+} f_{2}=\alpha,
$$

then, for any $\varepsilon>0$, there exists an $f_{3} \in C^{\infty}\left(\mathbb{R}^{n}\right)$ with

$$
\operatorname{supp} f_{3} \subset N_{\varepsilon}\left(\Omega_{1} \cap \Omega_{2}\right)
$$

and

$$
F_{q}^{+} f_{3}=\alpha .
$$

Proof. According to Rellich's lemma and unique continuation [3], $u_{1}=G_{q}^{+} f_{1}$ and $u_{2}=G_{q}^{+} f_{2}$ agree on the $\mathbb{R}^{n} \backslash\left(\Omega_{1} \bigcup \Omega_{2}\right)$.

Let $\phi \in C^{\infty}\left(\mathbb{R}^{n}\right)$ satisfy

$$
\phi= \begin{cases}1, & x \in \mathbb{R}^{n} \backslash N_{\varepsilon}\left(\Omega_{1} \cap \Omega_{2}\right), \\ 0, & x \in N_{\frac{\varepsilon}{2}}\left(\Omega_{1} \cap \Omega_{2}\right)\end{cases}
$$

then

$$
v= \begin{cases}\phi u_{1}, & x \in \mathbb{R}^{n} \backslash \Omega_{1}, \\ \phi u_{2}, & x \in \mathbb{R}^{n} \backslash \Omega_{2}, \\ 0, & x \in \Omega_{1} \cap \Omega_{2}\end{cases}
$$

is a well-defined $C^{\infty}$ function and $v=u_{1}=u_{2}$ outside a compact set, so that

$$
f_{3}=\left(\Delta+k^{2}-q(x)\right) v
$$

must also have far field $\alpha$. 
Lemma 4.5. For any $\epsilon>0$ and any far field $\alpha$ with a compactly supported source, there exists an integer $N$ and a sequence of sources $f_{n}$ such that

$$
N_{\epsilon}\left(\operatorname{cS}_{k} \operatorname{supp}_{q} \alpha\right) \supset \bigcap_{n=1}^{N} \operatorname{ch} \operatorname{supp}\left(f_{n}\right) \text {. }
$$

Proof. Let $B$ denote the complement of $\mathrm{cS}_{k} \operatorname{supp}_{q} \alpha$, let $B_{\epsilon}$ denote the complement of $N_{\epsilon}\left(\mathrm{cS}_{k} \operatorname{supp}_{q} \alpha\right)$, and let $A_{f}$ denote the complement of $\operatorname{ch} \operatorname{supp}(f)$. The $A_{f}$ 's are open and $B_{\epsilon}$ is closed. Taking complements in the definition (4.1) tells us that

$$
B=\bigcup_{F_{q}^{+} f=\alpha} A_{f} .
$$

We will prove the theorem by showing that we may choose $f_{n}$ such that

$$
B_{\epsilon} \subset \bigcup_{n=1}^{N} A_{f_{n}} .
$$

Let $f_{1}$ be a compactly supported source which radiates $\alpha$. Now $B_{\epsilon} \backslash A_{f_{1}}$ is compact and the $A_{f}$ 's provide an open cover of that compact set, so a finite subcover exists. Numbering that finite subcover $A_{f_{2}}$ through $A_{f_{N}}$ establishes (4.2) and proves the theorem.

Proof of Theorem 4.3. Lemma 4.5 implies that $N_{\epsilon}\left(\mathrm{cS}_{k} \operatorname{supp}_{q} \alpha\right)$ is contained in the intersection of finitely many sources. We may take $\Omega_{1}$ and $\Omega_{2}$ in Lemma 4.4 to be the convex hulls of the supports of two of the sources, so that the hypothesis that $\mathbb{R}^{n} \backslash\left(\Omega_{1} \bigcup \Omega_{2}\right)$ is connected is automatic. Thus we can produce a source supported on a neighborhood of the intersection of the convex hulls of the supports of any two sources, and we complete the proof by induction.

5. Active sensing: Finding the support of a scatterer. The convex scattering support of a far field which was not radiated by a source, but rather scattered by an inhomogeneity in a homogeneous medium, detects the deviation of the index of refraction from that of the homogeneous background medium. That is, when we illuminate the medium with an incoming wave, the inhomogeneity becomes a secondary, or induced, source, and our test can be applied to locate that source.

Similarly, we can apply the Picard test in an inhomogeneous background if we wish to locate the deviation of the index of refraction from that known background. In both cases, we apply the test to the deviation of the measured outgoing far field from the outgoing far field that we should have measured if no deviation were present. If the background is homogeneous, the test is applied to the scattered wave, the outgoing far field minus the antipodal map of the incoming field, which is the outgoing far field of the free solution with the same incoming far field. In the case of an inhomogeneous background, we subtract the wave scattered by the background.

In order to be mathematically precise we need to recall the scattering operator. We may formulate the scattering problem as

$$
\begin{gathered}
\left(\Delta+k^{2}-q(x)\right) u=0, \\
u_{\infty}^{-}(\Theta)=\beta(\Theta),
\end{gathered}
$$


where $\beta \in L^{2}\left(S^{n-1}\right)$ parameterizes the incoming far field (recall (2.13)). It is customary to seek the total wave $u$ as the sum of an incident wave and a scattered wave:

$$
\begin{aligned}
u & =u_{i n c}+u_{s c} \\
& =\mathcal{H} \beta+u_{s c} .
\end{aligned}
$$

In our notation, the incident wave is just the Herglotz operator (2.8) acting on $\beta$. Because $\mathcal{H} \beta$ has incoming far field equal to $\beta$, the scattered wave is outgoing and satisfies

$$
\begin{gathered}
\left(\Delta+k^{2}-q(x)\right) u_{s c}=q \mathcal{H} \beta, \\
\left(u_{s c}\right)_{\infty}^{-}=0 .
\end{gathered}
$$

Thus,

$$
u_{s c}=G_{q}^{+} q \mathcal{H} \beta
$$

and has far field

$$
\left(u_{s c}\right)_{\infty}^{+}=F_{q}^{+} q \mathcal{H} \beta
$$

The scattering operator, which maps the incoming far field to the scattered far field, is given by

$$
\begin{aligned}
S_{q} & =F_{q}^{+} q \mathcal{H} \\
& =F_{0}^{+} q\left(I-G_{0}^{+} q\right)^{-1} \mathcal{H} .
\end{aligned}
$$

If we can measure the far field $S_{q} \beta$ for a single incoming wave $\beta$, we may apply the Picard test to $S_{q} \beta$ to find what must be a subset of the convex hull of the support of the induced source

$$
\begin{aligned}
f & =q\left(I-G_{0}^{+} q\right)^{-1} \mathcal{H} \beta \\
& =q u .
\end{aligned}
$$

Because the unique continuation principle guarantees that $u$ cannot vanish on an open set, we can be certain that we are truly estimating the support of $q$, i.e., we have the following lemma.

LEMMA 5.1.

$$
\operatorname{supp} q u=\operatorname{supp} q .
$$

Suppose now that we are looking to locate not the support of $q$, but the support of $q-q_{b g}$. That is, we want to find the places where the medium deviates from the known inhomogeneous background $q_{b g}$. We rewrite (5.1) as

$$
\begin{gathered}
\left(\Delta+k^{2}-q_{b g}\right) u_{s c}=q_{b g} \mathcal{H} \beta+\left(q-q_{b g}\right) u, \\
\left(u_{s c}\right)_{\infty}^{-}=0,
\end{gathered}
$$

which shows that

$$
u_{s c}=G_{q_{b g}}^{+} \mathcal{H} \beta+G_{q_{b g}}^{+}\left(q-q_{b g}\right) u
$$


with outgoing far field

$$
S_{q} \beta=S_{q_{b g}} \beta+F_{q_{b g}}^{+}\left(q-q_{b g}\right) u .
$$

Thus, if we apply the Picard test to the far field

$$
S_{q} \beta-S_{q_{b g}} \beta
$$

we obtain an estimate of the support of the induced source $\left(q-q_{b g}\right) u$, which, according to Lemma 5.1, is a lower bound on the support of $q-q_{b g}$. We state this as a corollary of Theorem 3.6.

TheOREM 5.2. For every incident field $\alpha$,

$$
\mathrm{CS}_{k} \operatorname{supp}_{q}\left(S_{q_{b g}}^{+}-S_{q}^{+}\right) \alpha \subset \operatorname{ch} \operatorname{supp}\left(q-q_{b g}\right),
$$

i.e., if $q=q_{b g}$ in $\mathbb{R}^{n} \backslash \Omega$,

$$
\left(S_{q_{b g}}^{+}-S_{q}^{+}\right) \alpha \in \operatorname{Range}\left(\left.F_{q_{b g}}^{+}\right|_{L^{2}(\Omega)}\right)
$$

for every incident field $\alpha$.

6. Computing the convex scattering support. In the previous section, we have shown how to unambiguously associate a closed convex set, the convex scattering support, with a far field; we showed that any source which produces that far field must contain that set in the convex hull of its support, and that there always exists a source supported in any neighborhood of the convex scattering support which radiates that far field.

Theorem 3.6 tests whether that set is contained in a test region $\Omega$. In this section we describe a simple algorithm to make use of Theorem 3.6 to find the convex scattering support and show a numerical result for a homogeneous background in two dimensions to illustrate how the method works. We do not intend to suggest that what we present below is careful numerical study. It is meant to be illustrative. We do, however, view it as strong evidence that this provides a stable numerical method.

Algorithm. We will choose as test domain, $\Omega=B_{R}(c)$, the ball of radius $R$ with center $c$.

1. Choose the center $c=0$ and find the smallest $R$ such that $B_{R}(0)$ contains the scattering support. For a homogeneous background medium this is easily seen by simply plotting the modulus of the Fourier coefficients of the far field and looking for the place they become effectively zero (i.e., uniformly small). In the plot on the bottom left in Figure 1, the modulus of the Fourier coefficients are effectively zero for $|n|$ a little bigger than 50 . The wavenumber in this example is $k=50$, so the radius $R$ of the circle about zero is one $\left(\frac{50}{50}\right)$.

This succeeds because the decomposition of $\left.F_{0}^{+}\right|_{L^{2}\left(B_{c}(R)\right)}$ is exactly

$$
\left.F_{0}^{+}\right|_{L^{2}\left(B_{0}(R)\right)}=\sum_{n=-\infty}^{\infty} \mathrm{e}^{i n\left(\theta-\frac{\pi}{2}\right)} \otimes \chi_{B_{0}(R)}(r) J_{n}(k r) \mathrm{e}^{-i n\left(\phi-\frac{\pi}{2}\right)}
$$

(here $\chi_{B_{0}(R)}(r)$ is the characteristic function of the ball) so that its singular 

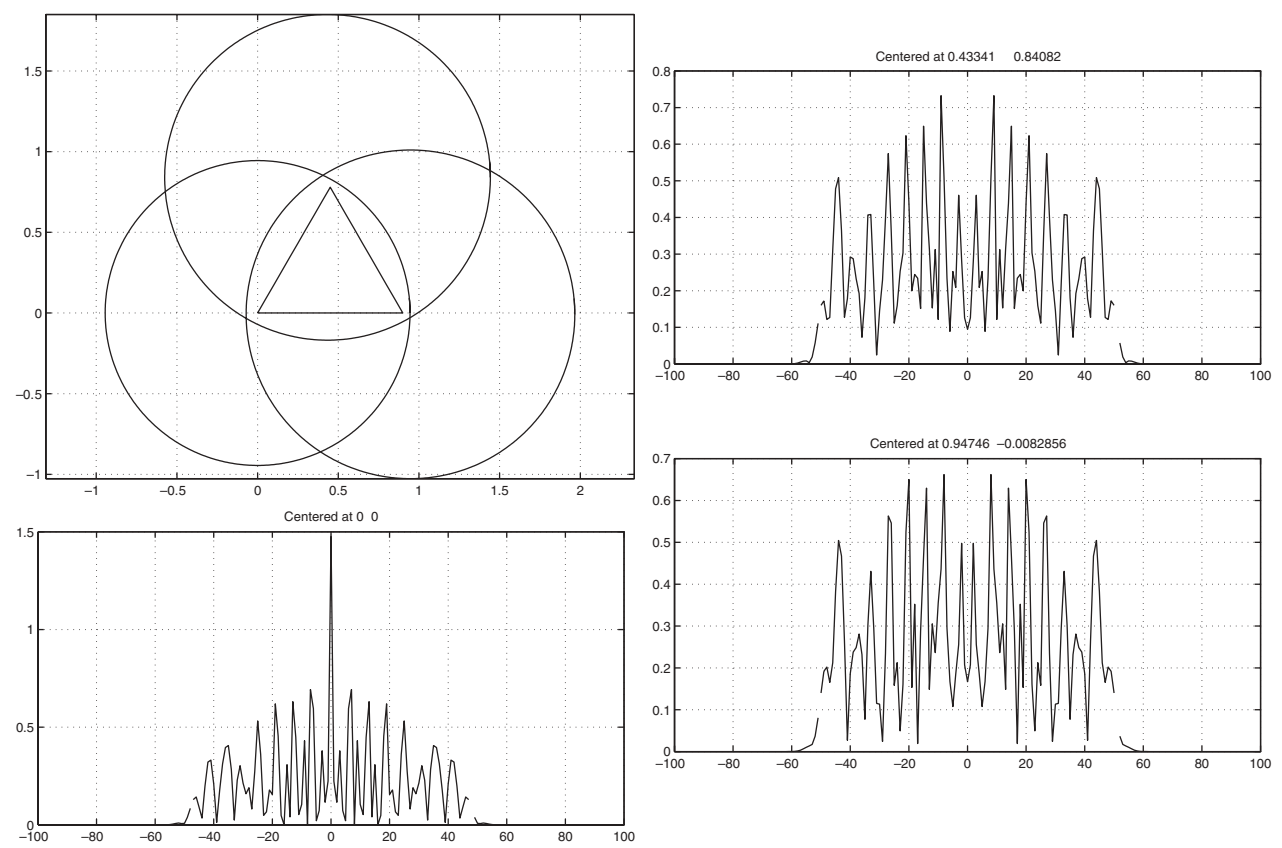

FIG. 1. Estimating the convex scattering support of a triangular source.

values are

$$
\begin{aligned}
\sigma_{n} & =\left(\int_{0}^{R} J_{n}^{2}(k s) s d s\right)^{\frac{1}{2}} \\
& \sim \begin{cases}\left(R^{2}-n^{2}\right)^{\frac{1}{4}} & \text { for } n<k R, \\
\frac{1}{\sqrt{n}}\left(\frac{\mathrm{e} R}{2 n}\right)^{n} & \text { for } n>k R,\end{cases}
\end{aligned}
$$

which means that the $\sigma_{n}$ are uniformly large for $n<k R$ and decay rapidly to zero as soon as $n>k R$.

2. Choose another center and repeat. In the homogeneous case, we compute the far field of the translated source instead of translating the test region. We use the formula

$$
F_{0}^{+} f(x-c)=\mathrm{e}^{i k|c| \cos \left(\theta-\theta_{c}\right)} F_{0}^{+} f
$$

and then apply the test from the previous step to the new far field given by the left-hand side of (6.2). The plots on the left-hand sides of Figures 1 and 2 are the results of translating the far fields to the centers indicated in the figures.

We don't suggest a specific algorithm for choosing the centers here. In the first example, we chose a new center to be on the intersection of previous circles. In the second, we needed to choose centers far from the line source to see that it was flat. One expensive alternative is just to grid space and then choose centers at each grid point.

3. Our estimate of the convex scattering support is the intersection of these balls as in the large plots at the top left of Figures 1 and 2). 

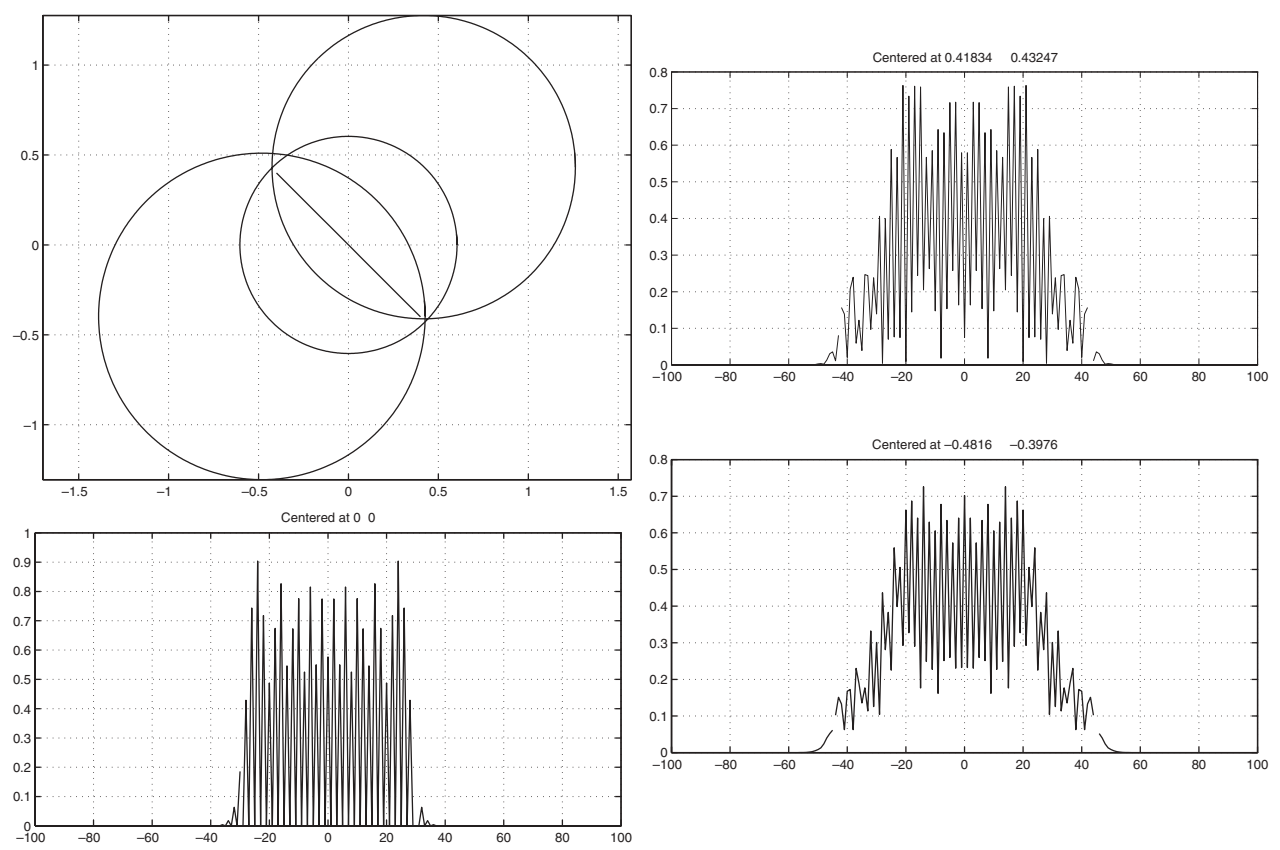

FIG. 2. Estimating the convex scattering support of a line source.

7. Conclusions. We have shown that the notion of the convex scattering support extends to scattering in an inhomogeneous background. Theoretically, the notion is actually quite general, relying only on unique continuation, and we expect it to hold in a very general mathematical setting. The observation of a far field can easily be replaced by the observation of a set of Cauchy data on all or part of the boundary of a region.

From a practical point of view, it is the threshold behavior of the $\sigma_{n}$ 's in (6.1) that is the most encouraging and intriguing. Equation (6.1) tells us not only that the Fourier coefficients of a far field produced in the ball of radius $R$ will go to zero rapidly when $n$ becomes greater than $k R$, but that they can, in general, ${ }^{1}$ be expected to be uniformly large for $n$ even slightly less than $k R$. This means that we need only look for this transition to zero, which is much less sensitive to noise than any sort of convergence or ratio test.

This threshold is intimately associated with wave propagation, and not merely a consequence of unique continuation. While the convex scattering support can easily be defined for Laplace's equation (the Helmholtz equation with $\omega=0$ ), the corresponding $\sigma_{n}$ 's exhibit no such behavior.

From our point of view, one very relevant question is whether this thresholding behavior occurs for other test domains and for Helmholtz equations with inhomogeneous backgrounds. If it does, we can expect these tests to be robust in the presence of noise as well.

\footnotetext{
${ }^{1}$ Theorem 3.6 itself guarantees that we can always artificially choose examples where the first 1000 Fourier coefficients are zero, and only after that do the hypotheses of the theorem hold. Nevertheless, we expect that, for a broad class of sources, this won't be the case.
} 


\section{REFERENCES}

[1] S. Agmon, Spectral properties of Schrödinger operators and scattering theory, Ann. Scuola Norm. Sup. Pisa Cl. Sci. (4), 2 (1975), pp. 151-218.

[2] D. Colton, J. Coyle, And P. Monk, Recent developments in inverse acoustic scattering theory, SIAM Rev., 42 (2000), pp. 369-414.

[3] D. Colton and R. Kress, Inverse Acoustic and Electromagnetic Scattering Theory, SpringerVerlag, Berlin, 1998.

[4] Y. V. Egorov and M. A. Shubin, eds., Partial Differential Equations I, Encyclopaedia Math. Sci. 30, Springer-Verlag, Berlin, 1991.

[5] M. Ikehata, On reconstruction in the inverse conductivity problem with one measurement, Inverse Problems, 16 (2000), pp. 785-793.

[6] M. Ikenata, A regularized extraction formula in the enclosure method, Inverse Problems, 18 (2002), pp. 435-440.

[7] A. KIRSCH, Factorization of the far-field operator for the inhomogeneous medium case and an application in inverse scattering theory, Inverse Problems, 15 (1999), pp. 413-429.

[8] S. Kusiak and J. Sylvester, The scattering support, Comm. Pure Appl. Math., 56 (2003), pp. $1525-1548$.

[9] D. R. Luke and R. Potthast, The no response test-a sampling method for inverse scattering problems, SIAM J. Appl. Math., 63 (2003), pp. 1292-1312.

[10] R. Potthast, J. Sylvester, And S. Kusiak, A "range test" for determining scatterers with unknown physical properties, Inverse Problems, 19 (2003), pp. 533-547.

[11] M. E. TAYLOR, Partial Differential Equations II: Qualitative Studies of Linear Equations, Appl. Math. Sci. 116, Springer-Verlag, New York, 1996. 